\title{
The Development of a 20MW PWM Driver for Advanced Fifteen-Phase Propulsion Induction Motors
}

\author{
Chi Sun ${ }^{\dagger}$, Sheng Ai*, Liangdeng $\mathrm{Hu}^{*}$, and Yulin Chen ${ }^{*}$ \\ $\dagger^{*}$ National Key Laboratory of Science and Technology on Vessel Integrated Power System, Naval University of \\ Engineering (NUE), Wuhan, China
}

\begin{abstract}
Since the power capacity needed for the propulsion of large ships is very large, a multiphase AC induction propulsion mode is generally adopted to meet the higher requirements of reliability, redundancy and maintainability. This paper gives a detailed description of the development of a 20MW fifteen-phase PWM driver for advanced fifteen-phase propulsion induction motors with a special third-harmonic injection in terms of the main circuit hardware, control system design, experiments, etc. The adoption of the modular design method for the main circuit hardware design can make the enclosed mechanical structure simple and maintainable. It can also avoid the larger switch stresses caused by the multiple turn on of the IGBTs in conventional large-capacity converter systems. The use of the distributed controller design method based on a high-speed fiber-optic ring net for the control system can overcome such disadvantages as the poor reliability and long maintenance times arising from the conventional centralized controller which is designed according to point-to-point communication. Finally, the performance of the 20MW PWM driver is verified by experimentation on a new fifteen-phase induction propulsion motor.
\end{abstract}

Key words: 20MW-level, Fifteen-phase, Hydraulic Dynamometer, Propulsion Motor, PWM Driver

\section{INTRODUCTION}

The continuous improvement of high-power power electronics techniques has promoted a rapid development of large-capacity adjustable-speed driver (ASD) techniques, which has greatly contributed to further research and development of large-capacity full-electric propulsion systems. These days, more and more ships are using or will begin to use electric propulsion instead of conventional mechanical propulsion systems. When compared with conventional mechanical propulsion systems, electric propulsion has the advantages of high efficiency, good flexibility, great maintainability and low fuel cost. At present, most of the electric propulsion systems used in commercial ships are based on synchronous motor driver systems with a cyclo-converter or load commutated inverter [1]. In addition to the above benefits,

Manuscript received Jun. 6, 2014; accepted Aug. 28, 2014

Recommended for publication by Associate Editor Dong-Hee Lee

†Corresponding Author: sunchi77@sina.com.cn

Tel: +86-136-6727-0090, Fax: +86-027-836-01907, NUE

"National Key Laboratory of Science and Technology on Vessel

Integrated Power System, Naval Univ. of Eng. (NUE), China the effective reduction of the vibration and noise of propulsion systems by applying electric propulsion to warships can raise their vitality and combat capability. Therefore, more and more attention has been paid to the development and application of military fully-electric propulsion techniques. For example, the British Royal Navy has employed an integrated fully-electric propulsion and the U.S. Navy has carried out an integrated power system (IPS) plan. Because driver systems based on a PWM voltage inverter are better than driver systems based on a cyclo-converter or a load commutated inverter in terms of power factor, waveform quality and smooth rotational speed transient process [2], they are often used in warships. As far as propulsion motors are concerned, AC induction motors are characterized by simple structure, mature manufacturing techniques and convenient maintenance when compared with AC synchronous motors, permanent-magnet motors and high temperature superconducting motors [3]. As a result, the application of AC induction motors to warships is a practical and feasible approach [1].

Since the propulsion of large ships has higher requirements in terms of reliability, redundancy and maintainability, 


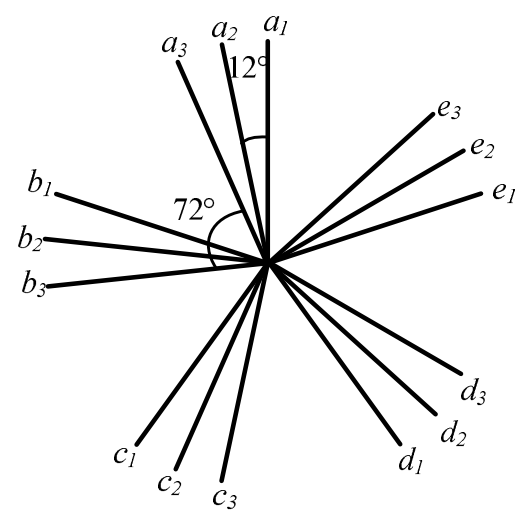

Fig. 1. The winding distribution of the advanced fifteen-phase $\mathrm{AC}$ induction motor.

multiphase AC induction propulsion systems are generally used for propulsion [4], [5]. Of course, various types of ships have different demands when it comes to the propulsion system power level. These demands may range from several MWs to tens of MWs. Normally, 20MW AC induction propulsion systems are very suitable for large surface ships, and a 40MW power rating can be gained by connecting two of these converters in parallel or by connecting two coaxial motors in series. Thus, they can satisfy the needs of electric propulsion for larger ships.

At present, five-phase and six-phase induction motors with medium and small capacities have become the focus of studies related to the ASDs of multiphase AC induction motors [6]-[9]. However, there are less reports on the study of propulsion converters with more phases and larger capacities [10]-[14]. This paper has presented a detailed description of the research and development of a 20MW PWM driver for advanced fifteen-phase propulsion induction motors with a special third-harmonic injection. It presents the designs of the main circuit hardware and control system as well as experiments. This can serve as a reference for the study of correlative large-capacity converters.

\section{SYSTEM DESCRIPTION}

A 20MW PWM driver is designed for advanced fifteen-phase open-winding AC induction propulsion motors with a special third-order harmonic injection. The aim of this driver is to improve the electromagnetic performance of these motors and to increase the torque density and operating redundancy [15], [16]. The winding distribution of an advanced of fifteen-phase AC induction motor is shown in Fig. 1. The angle difference among the three groups and that among the different windings, in the same group are $12^{\circ}$ and $72^{\circ}$ [17], respectively. The phase voltage of the motor winding, the rated rotational speed and the rated operating frequency are $2400 \mathrm{~V}$ (RMS), 200RPM and 20Hz, respectively.

The main circuit diagram of the 20MW PWM driver is shown in Fig. 2. The AC voltage from a $10 \mathrm{kV}$ electric network is supplied to three groups of five-phase H-bridge inverter units after being stepped down and rectified by three $3 / 6$ phase transformers with primary windings mutually differing by $10^{\circ}$ in terms of phase shift. While considering the output waveform quality, the neutral-point-clamped (NPC) five-level H-bridge main circuit topology is applied to every inverter unit, and a single NPC five-level $\mathrm{H}$ bridge inverter unit for the $20 \mathrm{MW}$ PWM driver is mainly composed of 4 diode modules, 8 IGBT modules and drivers, where the DYNEX 3300V/1200A diode is used in the diode module, and the DYNEX $3300 \mathrm{~V} / 1500 \mathrm{~A}$ IGBT and CONCEPT's 1SD536F2 are used in the IGBT module and driver, respectively. The DC power for every group of five-phase H-bridge inverter units is supplied by a series-connection twelve-pulse rectifier with a midpoint output. In this way, the neutral-point voltage balance of the NPC five-level $\mathrm{H}$ bridge inverter units can be guaranteed to be reliable. In addition, the equivalent with 36-pulse rectified current waveforms can be formed at the $\mathrm{AC}$ input side of the $10 \mathrm{kV}$ electric network, which greatly reduces the pollution of the $10 \mathrm{kV}$ electric network with harmonic. In order to implement the quick brake of the propulsion induction motor, three brake units are designed for the PWM driver to absorb the regenerated energy of the motor.

The topology of Fig. 2 is widely used in the civil field. It needs several $3 / 6$ phase transformers and rectifiers since a mismatch exists between the voltage of the electric network and the input voltage of the PWM driver. While the transformers are not needed in the military field since the power capacity of the electric propulsion system exceeds $90 \%$ of the whole vessel's and the PWM driver is usually powered by generators which also provide electrical power for daily service loads in vessel integrated power systems [18]. In order to increase the power density of the whole propulsion system, the main circuit structure of the propulsion system can be used, as shown in Fig. 3. The common DC bus provided by one or several integrated 12-phase synchronous rectifier-generators can be used to supply electricity to both the propulsion system and daily loads. Thus, three large step-down transformers are saved and the system power density is greatly enhanced.

\section{THE MOdUlar Design OF THE MAIN CIRCUIT}

At present, the centralized hardware design method is usually adopted for designing main circuits, with the DC link capacitors and power switches arranged centrally and the low-inductance laminated bus bar connected electrically.

In the circuit topology shown in Fig. 3, the adoption of the centralized main circuit hardware design method makes it difficult to design and produce the laminated bus bar. This results in poor maintainability of the main circuit due to the large number of power devices and the large volume of the DC connecting capacitors [19]. Moreover, the centralized design will lead to a large common stray inductance and extra 


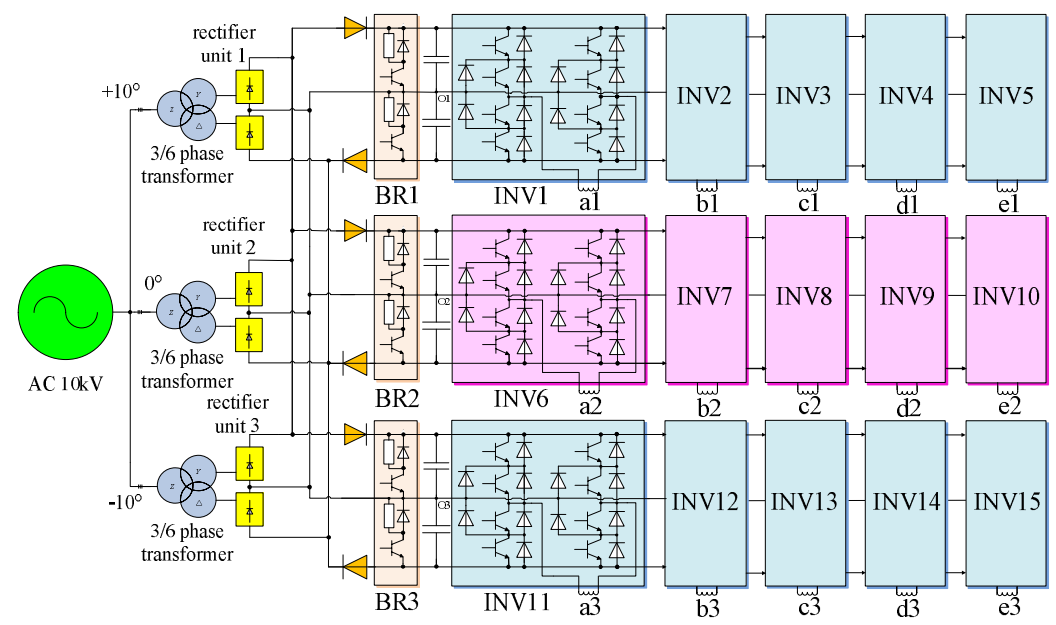

Fig. 2. The topology of the 20MW fifteen-phase PWM driver.

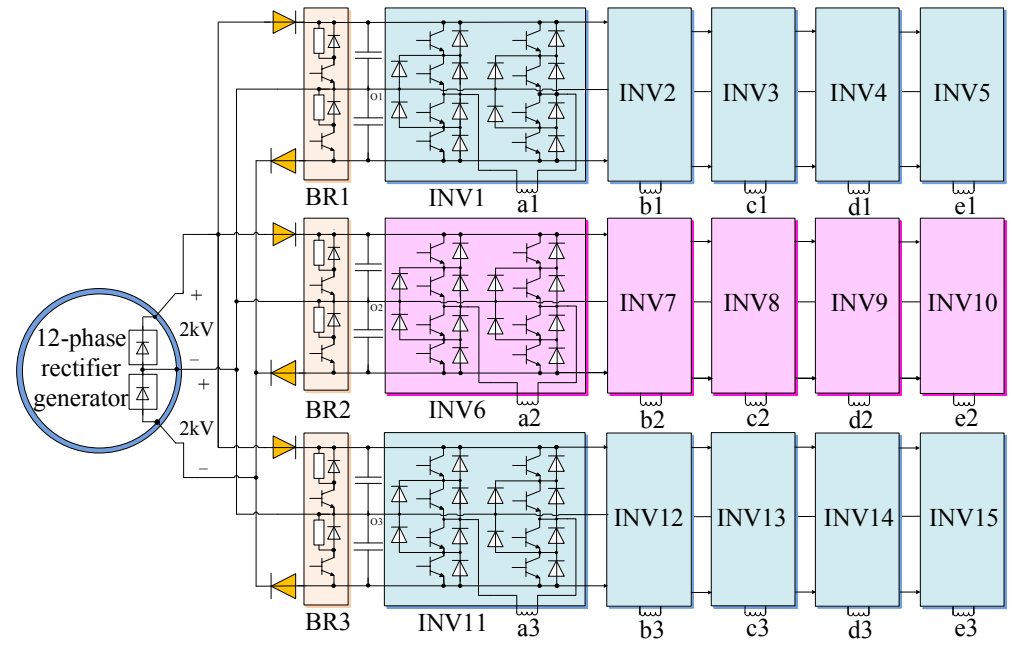

Fig. 3. The 20MW PWM driver with dc bus voltage generated by 12-phase rectifier generator.

switching stress. An equivalent circuit diagram of the switching stress which is generated and shown in Fig. 4 [20], [21]. Because of the existence of a common stray inductance $L_{\text {common }}$, when multiple IGBTs in different phases are switching synchronously or almost synchronously, a switch commutating voltage spike appears in the IGBT. In addition, its anti-parallel diode in the $i$ phase is related to the stray inductance $L_{i}$ and $d i / d t$ of the $i$ phase, and to the common stray inductance $L_{\text {common }}$ and the total dc bus $d i / d t\left(d i_{\Sigma} / d t\right)$. Furthermore, the stress of the switching devices will increase significantly in this operating mode. To solve this problem, reference [19] proposed two methods. One is to design a compact DC bus bar so as to reduce the common stray inductance; the other is to use active gate control techniques to control the commutating $d i / d t$. For medium-voltage large-capacity multilevel converters, the adoption of the design method for a centralized main circuit causes a lot of difficulty in the design of a compact DC bus bar, because the size and weight of the DC bus bar is very large, and the multi-layer bus bar needs to be designed with higher insulation performance taken into account. Active gate control techniques can decrease the switching stress of the switching devices. Designing an active gate driving circuit capable of reducing switch loss and matching the main circuit parameters is worthy of deep study.

In the operating conditions of the motor excitation and transient process, multiple IGBTs in different phases can switch on and off at the same time or at nearly at the same time in the 20MW fifteen-phase propulsion converter. This creates high switching stress on the IGBT and especially on its anti-parallel diode. Therefore, this problem must be taken into account in the design of the main circuit. For this reason, a modular main circuit hardware design method has been adopted.

Fig. 5 shows an equivalent circuit based on the adopted modular main circuit hardware design for the $20 \mathrm{MW}$ fifteen-phase propulsion converter. The DC link capacitors are composed of many small DC capacitors, which are made to correspond to all of the brake units and NPC five-level $\mathrm{H}$-bridge inverter units. The common stray inductance of the DC bus bar can be fully decoupled by the DC link capacitor 


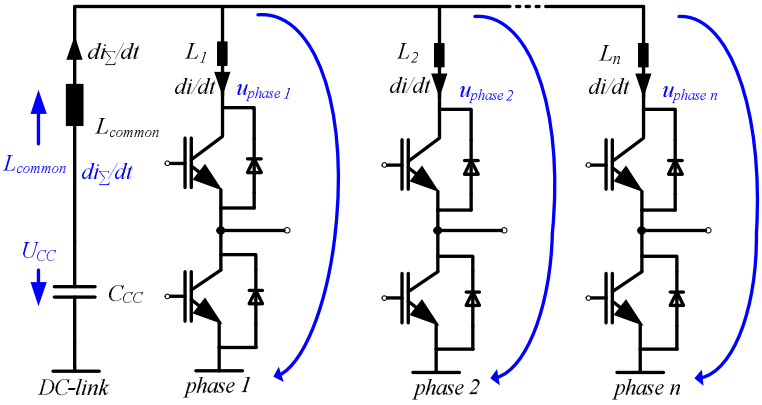

Fig. 4. Equivalent circuit diagram of the shared dc bus multiphase inverter with common stray inductance.

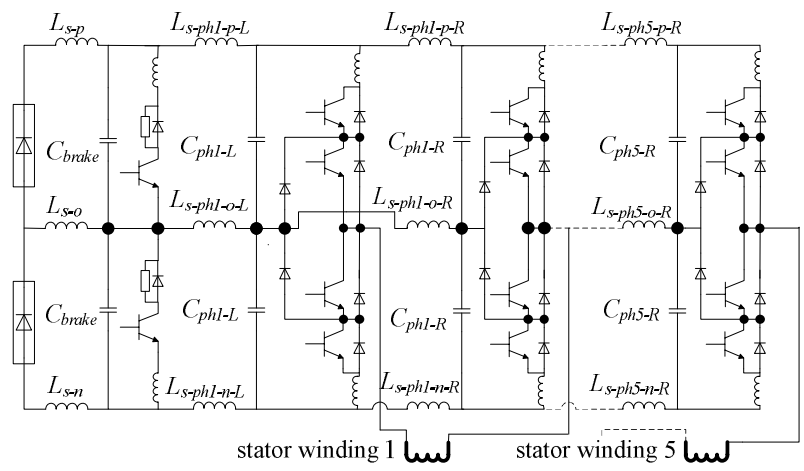

Fig. 5. The single-channel five-phase equivalent circuit designed with the modular main circuit hardware method.

(with a capacitance up to a $\mathrm{mF}$ rating) of all of the brake units or diode clamping five-level inverter bridge. Thus, a commutating loop design which only refers to all of the brake units or NPC five-level H-bridge inverter units can be greatly simplified. In addition, the use of a single copper bus bar to obtain the DC link bus bar can decrease the cost and increase maintainability [22], [23]. Because of the different stray coupling inductance between the DC capacitors, a low-frequency circulating current will appear to a certain extent. Then, the current rating of the DC capacitor will be a little larger than the real calculated value.

Fig. 6 shows transient load short-circuit test waveforms with the $4400 \mathrm{~V}$ DC bus voltage of a NPC five-level H-bridge inverter unit which is the farthest from the DC input. From these waveforms it can be seen that the voltage overshoot passing through the inner IGBT with the longest commutating path is about $670 \mathrm{~V}$ when the peak value of the switching-off current is $1730 \mathrm{~A}$. Therefore, during the commutating process, the two freewheeling diodes corresponding to the IGBT produces a forward recover voltage overshoot of about $230 \mathrm{~V}$ in the commutating process.

\section{THE DESIGN OF THE DisTRIBUTED CONTROL SYSTEM}

\section{A. Principle Description}

Nowadays, the centralized control method is generally adopted for various speed drivers whose typical control

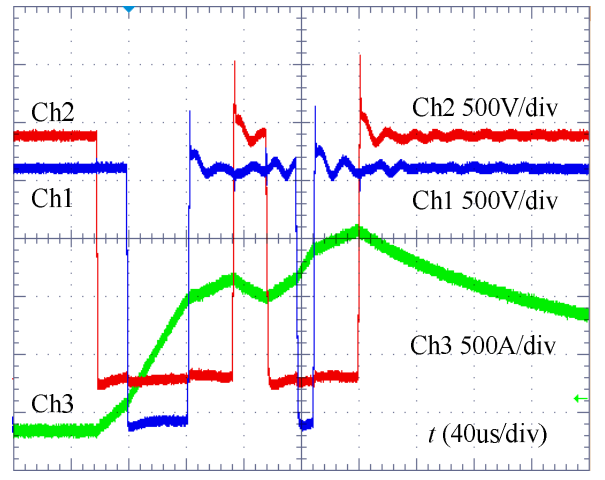

Fig. 6. Test waveforms of NPC five-level H-bridge inverter unit. (Ch1: Blue represents, the external switch voltage; Ch2: Red represents, the inner switch voltage; Ch3: Green represents, the load current)

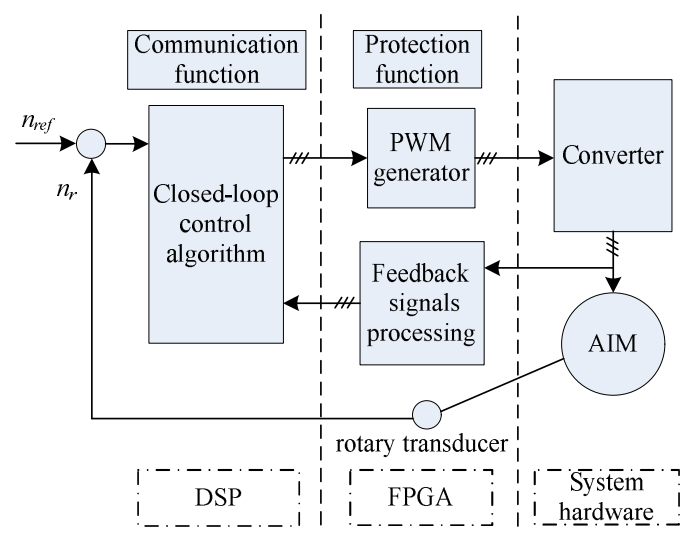

Fig. 7. The typical centralized control schematic diagram of ASDs.

functions and methods can be described by Fig. 7. The DSP, which has great calculating capability, is responsible for the implementation of the motor close-loop control algorithm. The FPGA, whose running speed is fast and which has abundant logic blocks and registers, performs the control functions closely related to the power devices, such as the digital processing of feedback signals, the modulation of PWM pulse width, and the protection of the system.

The development of the 20MW PWM driver for advanced fifteen-phase propulsion induction motors includes a large number of switch devices and feedback signals. If the centralized control design and point-to-point communication are adopted, there will an obvious decrease in the reliability and maintainability of the controller. In addition, the EMC performance of the controller will be difficult to secure. Thus, the redundancy of the whole propulsion system will be damaged. As shown in Fig. 3, the hardware function of the whole 20MW PWM driver is apparent in modularity. The adopted modular main circuit design method mentioned above makes it possible for the whole PWM driver to have the obvious characteristics of modularization. Therefore, it is an ideal choice to adopt a distributed control system design based on the high-speed serial fiber-optic ring net [24]. This can greatly increase the reliability, redundancy and fast recovery of 


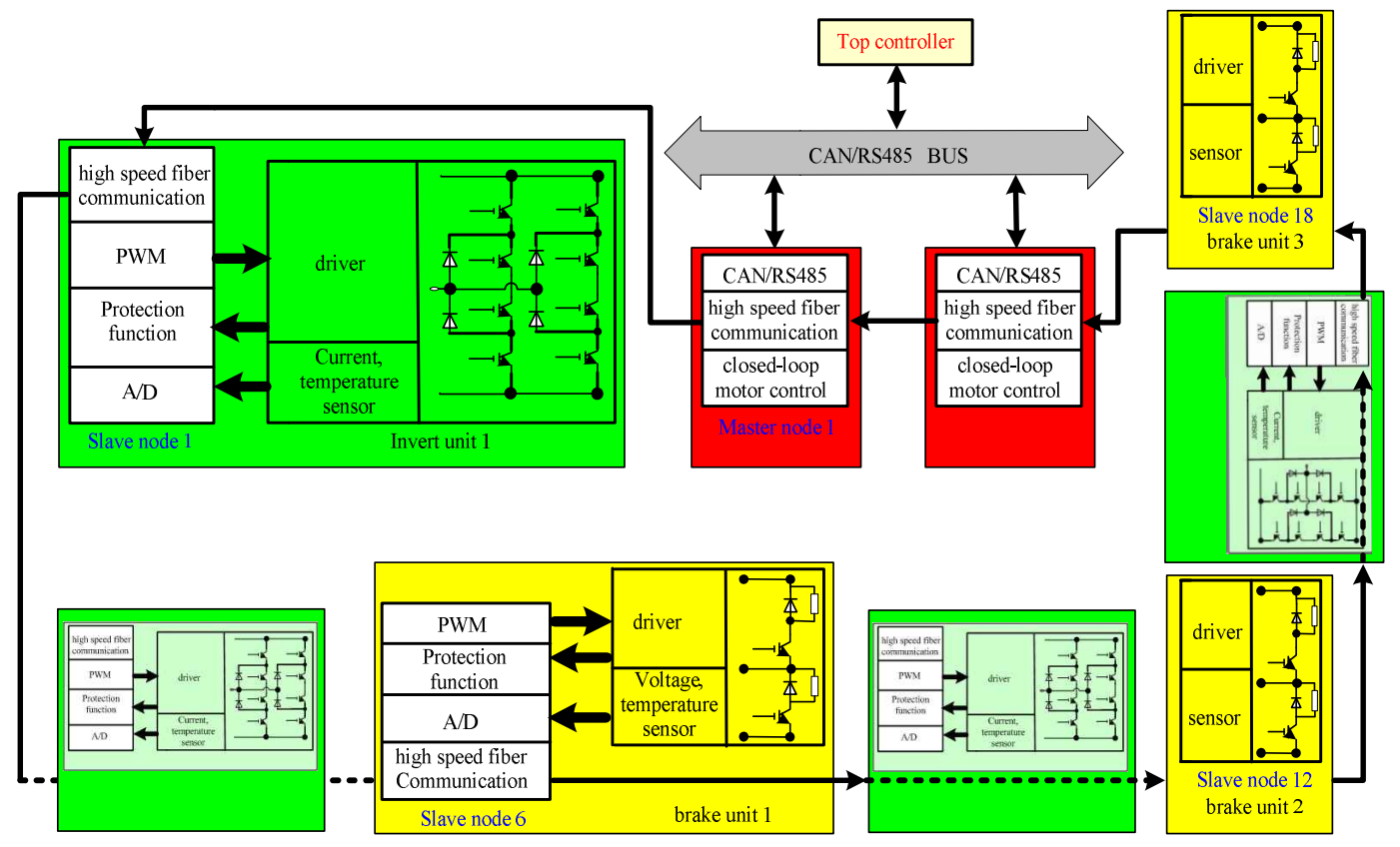

Fig. 8. The distributed control principle scheme of 20MW PWM driver.

the whole propulsion system under fault conditions. According to the principles of standardization and modularization, eighteen modules, or three brake units and fifteen NPC five-level H-bridge inverter units, serve as slave nodes. There are two master control nodes configured for the function backup. The whole high-speed serial fiber-optic ring net consisting of twenty nodes can reach a communication rate of $155 \mathrm{Mb} / \mathrm{s}$ according to the design. As a result, a standard industrial PECL transceiver can be employed. However, in practice, the communication of the prototype rate is $125 \mathrm{Mb} / \mathrm{s}$. According to the control requirements of the 20MW PWM driver for advanced fifteen-phase propulsion induction motors, the functions shown in Fig. 7 are decomposed, with one for each node. The distributed control principle scheme of the 20MW driver is shown in Fig. 8. From this figure, it can be seen that the hardware implements the function and spatial distribution in the control system. The corresponding software function is also assigned to the corresponding node controller, with the result that the function of the system software is of better modularization, which makes team programming easier.

The master controller is composed of a high-performance float-point DSP (TI TMS320F28335) and a high-density FPGA (Altera EP3C80F484). The DSP carries out the motor close-loop control algorithm and communicates with the upper controller, while the FPGA performs the send-receive control function of the high-speed fiber-optic ring net. The slave controller consisting of a single-chip high-density FPGA and the corresponding peripheral chips perform the send-receive control function of the high-speed fiber-optic ring net, the PWM control, the sampling and processing of the voltage, the current and temperature signals of the local main circuit unit,

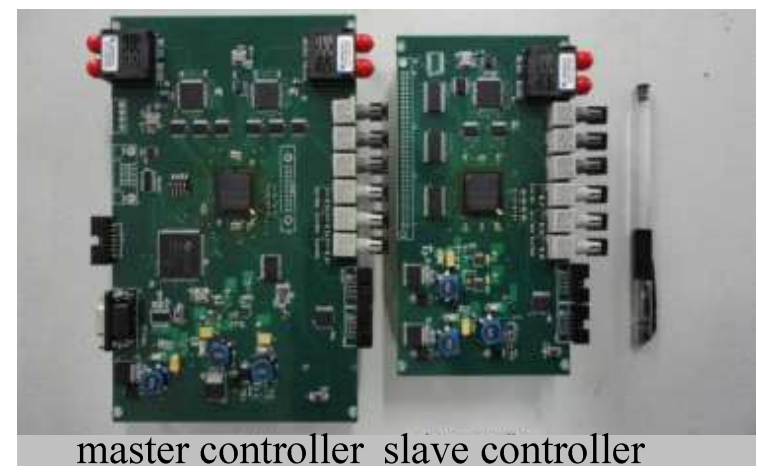

Fig. 9. The pictures of master and slave controllers.

and the fault protection function of the unit. Fig. 9 shows a picture of the master and slave controllers. The master controller is designed as one unit with two high-speed fiber-optic communication ports. Thus, it can be used to design the control system of the 20MW fifteen-phase PWM driver as a nested four-ring network structure, as shown in Fig. 10, which is composed of three bottom rings and a top ring. Each bottom ring consists of a group of five-phase H-bridge inverter units and a brake unit, as shown in Fig. 3, and the top ring consists of three master controllers, each in a bottom ring, for the purpose of increasing the reliability of the system. Fig. 11 shows the PECL signal waveform at a communication rate of $125 \mathrm{Mb} / \mathrm{s}$ measured at the port of the high-speed fiber-optic transceiver.

\section{B. Communication Protocol Description}

As shown in Fig. 8, the master node controller sends modulation-wave control data to each of the slave nodes in every switching period, and each of the slave nodes sends its 


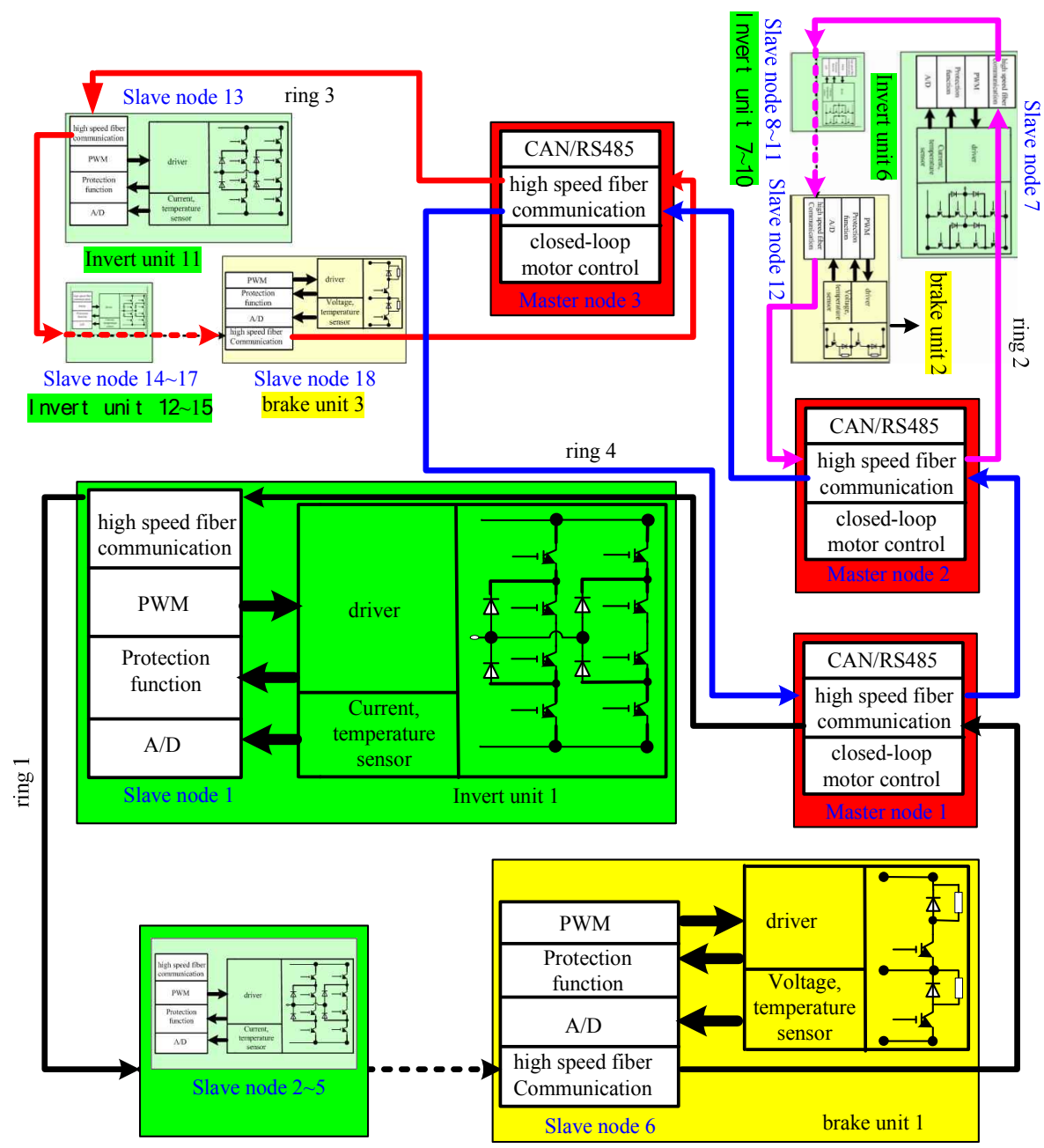

Fig. 10. The distributed-control principle scheme of 20MW PWM driver based on multiple-ring high-speed fiber-optic communication topology.

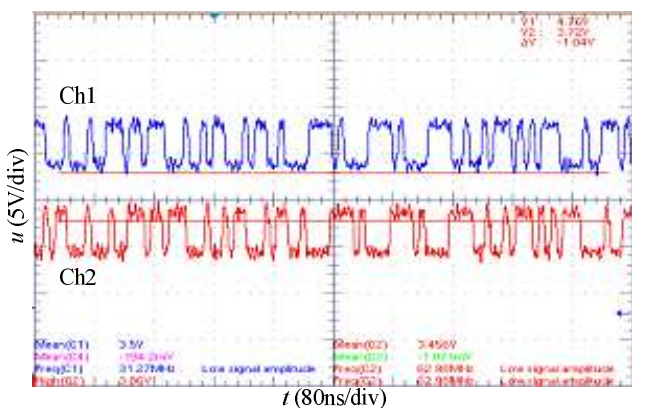

Fig. 11. The fiber-optic transceiver PECL signal waveform at the communication rate of $125 \mathrm{Mb} / \mathrm{s}$.

feedback data and operating-state information to the master controller. Since different nodes are connected by a single fiber, there is no definite sequential relationship between the slave nodes which are closely correlated to power devices. To ensure that the PWM control sequence is correct, different slave nodes must follow strict sequential synchronization. The realization of the PWM gate signal synchronization between inverter units is described as follows: The interrupt signal is generated at the

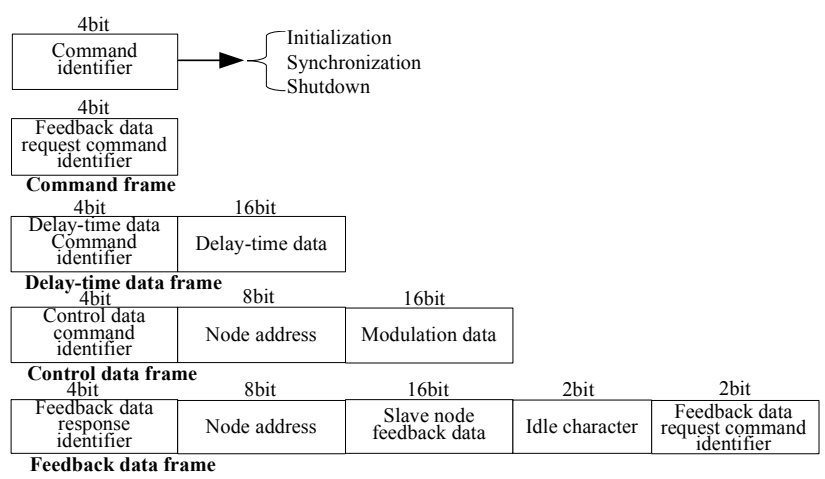

Fig. 12. Data formats

beginning of the switching period by the master controller. According to the interrupt signal, the synchronization command is sent from the master controller to slave controllers via the high-speed fiber-optic ring net. Moreover, in order to synchronize the switching timing of the slave controllers, an individual delay time is set to each slave controller based on its position in the ring net when the slave controller receives 


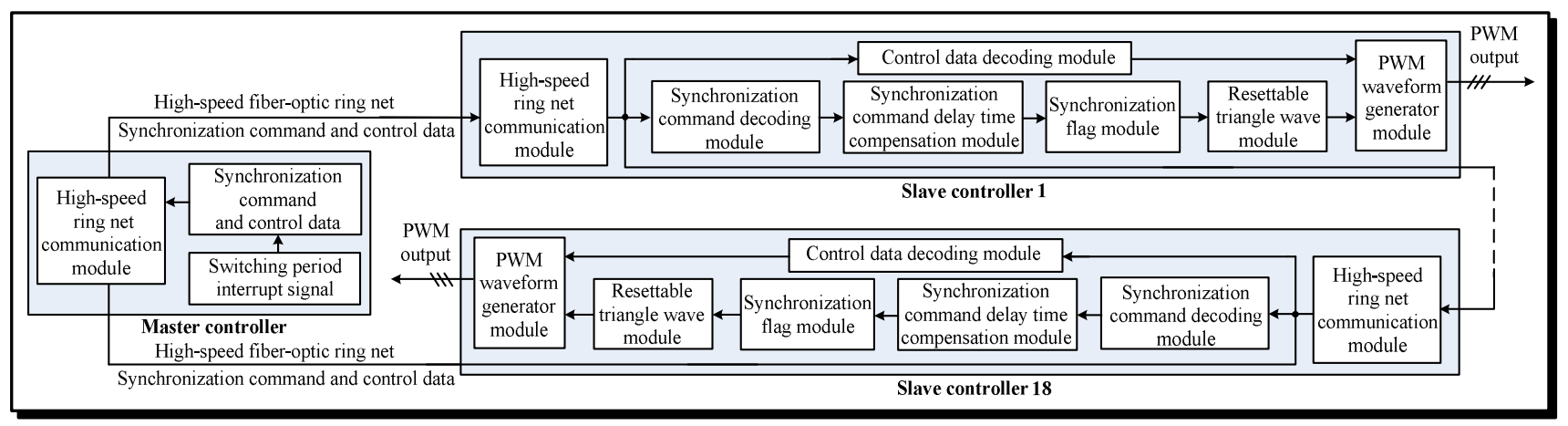

Fig. 13. Overall block diagram of synchronization and generation of PWM gate signals.

master controller synchronization command. Furthermore, a synchronization flag is generated by the slave controller after the compensation of the delay time is set. This is used as a resettable signal for the triangle wave module to prevent an accumulation error of the synchronous PWM gate signal. Finally, the triangular wave and control data received via the high-speed fiber-optic ring net are sent to the PWM waveform generator, which results in the desired PWM signals for the NPC five-level $\mathrm{H}$ bridge inverter units. An overall block diagram of the synchronization and generation of the PWM gate signals is shown in Fig. 13.

In addition, according to the above operational requirements, it is necessary to work out the corresponding data formats, which refer to the command frame, time-delay data frame, control data frame and feedback data frame, as shown in Fig.12. The four command and data frames are described below.

\section{1) Command Frame}

a) Initialization Command (Master to Slave): This is used to instruct each of the slave nodes to initialize its function. Then the delay time of the whole ring net is calculated according to the time difference in receiving and transmitting the initialization command of the master controller.

b) Synchronization Command (Master to Slave): After the synchronization command is received, the timing count function is started in accordance with the received delay data and node address, and the synchronization flag signal generated for the PWM is output when the timing process is over.

c) Shut-Down Command (Master to Slave): The whole propulsion converter should be shut down at the system's need.

d) Feedback Data Request Command (Master to Slave): The master controller sends the feedback data request command to each of the slave nodes in every switching period, and the slave node sends its feedback data and operating-state information to the master controller.

2) Data Frame

a) Delay-Time Data Frame (Master to Slave): The delay time data of the whole ring net that is measured by the master controller is transmitted to all of the nodes during the process of initialization.

b) Control Data Frame (Master to Slave): The modulation control data needed by each of the slave nodes is sent during every switch sampling period.

c) Feedback Data Frame (Slave to Master): When the first slave node receives the feedback data request command from the master controller, the node will first send the feedback data to the master controller in accordance with the format of "response command identifier - slave node address - feedback data of slave node." Then the node will send the feedback data request command again and the following two characters are idle. The downstream node just performs a simple data feedback forwarding function from the upstream node. However, it uploads its feedback data according to the format of "response command identifier - slave node address feedback data of slave node," only when it receives the feedback data request order.

Since the master controller only needs to send a 4-bit feedback data request command, it is possible to save the quantity of communication data greatly, shorten the communication time and reserved more time for the control algorithm, when compared with the methods proposed in the literature [25], [26]. Thus, the control algorithm of more complex and advanced systems can be convenient to use. Besides, in order to overcome communication errors, the function of the cyclic redundancy check (CRC) can be added in all of the command or data frames as shown in Fig.12. However, the CRC increases the length of the command and data. Therefore, in real applications, the indirect judgment method is adopted by comparing the delivered control data from the master controller with the returned control data via the ring net in each switching period. If a difference exists, a communication error has occurred.

\section{THE VECTOR CONTROL OF ADVANCED FIFTEEN-PHASE INDUCTION MOTORS}

Ships are often subjected to high waves and winds when sailing on the sea. When working in bad weather like this, the PWM driver must meet the higher requirements of dynamic performance. Therefore, the closed-loop vector control method is adopted for the new 20MW fifteen-phase induction motor PWM driver. Its simplified closed-loop vector control principle 
diagram is shown in Fig. 15. A brief derivation is given below.

Without taking the magnetic saturation and loss into consideration, the windings are assumed to have a sinusoidal distribution, with the resistance of each phase stator winding being equal. Then, the balance equation of the stator voltage and magnetic linkage of the advanced fifteen-phase induction motor is as follows:

$$
\left\{\begin{array}{l}
U_{\mathrm{s}}=R_{\mathrm{s}} I_{\mathrm{s}}+\frac{\mathrm{d} \psi_{\mathrm{s}}}{\mathrm{d} t} \\
\psi_{\mathrm{s}}=L_{\mathrm{ss}} I_{\mathrm{s}}+L_{\mathrm{sr}} I_{\mathrm{r}}
\end{array}\right.
$$

Where, $R_{\mathrm{s}}, I_{\mathrm{s}}, I_{\mathrm{r}}$, and $U_{\mathrm{s}}$ represent the matrixes of the stator resistance, stator current, rotor current and stator voltage matrix, respectively. Their expressions are shown as (2)-(5), respectively. In addition, $L_{\mathrm{ss}}$ and $L_{\mathrm{sr}}$ are the mutual inductance matrix between the stator windings and the mutual inductance matrix between the stator and rotor windings, respectively. The corresponding expressions are shown as (6)-(7), respectively.

$$
\begin{gathered}
I_{\mathrm{s}}=\left[\begin{array}{llllllll}
i_{\mathrm{a} 1 \mathrm{~s}} & i_{\mathrm{b} 1 \mathrm{~s}} & i_{\mathrm{c} 1 \mathrm{~s}} & i_{\mathrm{d} 1 \mathrm{~s}} & i_{\mathrm{e} 1 \mathrm{~s}} & i_{\mathrm{a} 2 \mathrm{~s}} & \ldots & i_{\mathrm{e} 3 \mathrm{~s}}
\end{array}\right]^{t} \\
I_{\mathrm{r}}=\left[\begin{array}{llllllll}
i_{\mathrm{a} 1 \mathrm{r}} & i_{\mathrm{b} 1 \mathrm{r}} & i_{\mathrm{c} 1 \mathrm{r}} & i_{\mathrm{d} 1 \mathrm{r}} & i_{\mathrm{e} 1 \mathrm{r}} & i_{\mathrm{a} 2 \mathrm{r}} & \ldots & i_{\mathrm{e} 3 \mathrm{r}}
\end{array}\right]^{t} \\
U_{\mathrm{s}}=\left[\begin{array}{llllllll}
u_{\mathrm{a} 1 \mathrm{~s}} & u_{\mathrm{b} 1 \mathrm{~s}} & u_{\mathrm{c} 1 \mathrm{~s}} & u_{\mathrm{d} 1 \mathrm{~s}} & u_{\mathrm{e} 1 \mathrm{~s}} & u_{\mathrm{a} 2 \mathrm{~s}} & \ldots & u_{\mathrm{e} 3 \mathrm{~s}}
\end{array}\right]^{t}(5) \\
L_{\mathrm{ss}}=\left[\begin{array}{ccccccc}
L_{\mathrm{a} 1 \mathrm{a} 1 \mathrm{~s}} & L_{\mathrm{a} 1 \mathrm{~b} 1 \mathrm{~s}} & \cdots & L_{\mathrm{a} 1 \mathrm{~d} 3 \mathrm{~s}} & L_{\mathrm{a} 1 \mathrm{e} 3 \mathrm{~s}} \\
L_{\mathrm{b} 1 \mathrm{a} 1 \mathrm{~s}} & L_{\mathrm{b} 1 \mathrm{~b} 1 \mathrm{~s}} & \cdots & L_{\mathrm{b} 1 \mathrm{~d} 3 \mathrm{~s}} & L_{\mathrm{b} 1 \mathrm{e} 3 \mathrm{~s}} \\
\cdots & \cdots & \cdots & \cdots & \cdots \\
L_{\mathrm{d} 3 \mathrm{a} 1 \mathrm{~s}} & L_{\mathrm{d} 3 \mathrm{~b} 1 \mathrm{~s}} & \cdots & L_{\mathrm{d} 3 \mathrm{~d} 3 \mathrm{~s}} & L_{\mathrm{d} 3 \mathrm{e} 3 \mathrm{~s}} \\
L_{\mathrm{e} 3 \mathrm{a} 1 \mathrm{~s}} & L_{\mathrm{e} 3 \mathrm{~b} 1 \mathrm{~s}} & \cdots & L_{\mathrm{e} 3 \mathrm{~d} 3 \mathrm{~s}} & L_{\mathrm{e} 3 \mathrm{e} 3 \mathrm{~s}}
\end{array}\right] \\
L_{\mathrm{sr}}=L_{\mathrm{rs}}{ }^{t}=\left[\begin{array}{ccccccc}
L_{\mathrm{a} 1 \mathrm{sa} 1 \mathrm{r}} & L_{\mathrm{a} 1 \mathrm{sb} 1 \mathrm{r}} & \cdots & L_{\mathrm{a} 1 \mathrm{sd} 3 \mathrm{r}} & L_{\mathrm{a} 1 \mathrm{se} 3 \mathrm{r}} \\
L_{\mathrm{b} 1 \mathrm{sa} 1 \mathrm{r}} & L_{\mathrm{b} 1 \mathrm{sb} 1 \mathrm{r}} & \cdots & L_{\mathrm{b} 1 \mathrm{sd} 3 \mathrm{r}} & L_{\mathrm{b} 1 \mathrm{se} 3 \mathrm{r}} \\
\cdots & & \cdots & \cdots & \cdots & \cdots \\
L_{\mathrm{d} 3 \mathrm{sa} 1 \mathrm{r}} & L_{\mathrm{d} 3 \mathrm{sb} 1 \mathrm{r}} & \cdots & L_{\mathrm{d} 3 \mathrm{sd} 3 \mathrm{r}} & L_{\mathrm{d} 3 \mathrm{se} 3 \mathrm{r}} \\
L_{\mathrm{e} 3 \mathrm{sa} 1 \mathrm{r}} & L_{\mathrm{e} 3 \mathrm{sb} 1 \mathrm{r}} & \cdots & L_{\mathrm{e} 3 \mathrm{sd} 3 \mathrm{r}} & L_{\mathrm{e} 3 \mathrm{se} 3 \mathrm{r}}
\end{array}\right]
\end{gathered}
$$

In the above formulas, $r_{\mathrm{s}}$ is the stator resistance, $I_{15 \times 15}$ is a $15 \times 15$ unit diagonal matrix, and the superscript $t$ represents the transposition.

Similarly, the balance equation of the rotor voltage and magnetic linkage can be expressed as formula (8).

$$
\begin{gathered}
\left\{\begin{array}{c}
U_{\mathrm{r}}=R_{\mathrm{r}} I_{\mathrm{r}}+\frac{\mathrm{d} \psi_{\mathrm{r}}}{\mathrm{d} t} \\
\psi_{\mathrm{r}}=L_{\mathrm{sr}}^{\mathrm{t}} I_{\mathrm{s}}+L_{\mathrm{rr}} I_{\mathrm{r}}
\end{array}\right. \\
R_{\mathrm{r}}=r_{\mathrm{r}} \times I_{15 \times 15}
\end{gathered}
$$

$$
L_{\mathrm{rr}}=\left[\begin{array}{ccccc}
L_{\mathrm{a} 1 \mathrm{a} 1 \mathrm{r}} & L_{\mathrm{a} 1 \mathrm{~b} 1 \mathrm{r}} & \cdots & L_{\mathrm{a} 1 \mathrm{~d} 3 \mathrm{r}} & L_{\mathrm{a} 1 \mathrm{e} 3 \mathrm{r}} \\
L_{\mathrm{b} 1 \mathrm{a} 1 \mathrm{r}} & L_{\mathrm{b} 1 \mathrm{~b} 1 \mathrm{r}} & \cdots & L_{\mathrm{b} 1 \mathrm{~d} 3 \mathrm{r}} & L_{\mathrm{b} 1 \mathrm{e} 3 \mathrm{r}} \\
\cdots & \cdots & \cdots & \cdots & \cdots \\
L_{\mathrm{d} 3 \mathrm{a} 1 \mathrm{r}} & L_{\mathrm{d} 3 \mathrm{~b} 1 \mathrm{r}} & \cdots & L_{\mathrm{d} 3 \mathrm{~d} 3 \mathrm{r}} & L_{\mathrm{d} 3 \mathrm{e} 3 \mathrm{r}} \\
L_{\mathrm{e} 3 \mathrm{a} 1 \mathrm{r}} & L_{\mathrm{e} 3 \mathrm{~b} 1 \mathrm{r}} & \cdots & L_{\mathrm{e} 3 \mathrm{~d} 3 \mathrm{r}} & L_{\mathrm{e} 3 \mathrm{e} 3 \mathrm{r}}
\end{array}\right]
$$

$$
U_{\mathrm{r}}=\left[\begin{array}{llllllll}
u_{\mathrm{a} 1 \mathrm{r}} & u_{\mathrm{b} 1 \mathrm{r}} & u_{\mathrm{c} 1 \mathrm{r}} & u_{\mathrm{d} 1 \mathrm{r}} & u_{\mathrm{e} 1 \mathrm{r}} & u_{\mathrm{a} 2 \mathrm{r}} & \ldots & u_{\mathrm{e} 3 \mathrm{r}}
\end{array}\right]^{t}(11)
$$

In (8)-(11) (8), $R_{\mathrm{r}}, L_{\mathrm{rr}}$, and $U_{\mathrm{r}}$ represent the stator resistance matrix, the mutual inductance matrix between the rotor windings, and the rotor voltage matrix, respectively. Their expressions are shown as (9)-(11), where, $r_{\mathrm{r}}$ is the rotor resistance.

The sinusoidal distribution of the magnetic field is the basis of the traditional $\mathrm{dq}$ coordinate transformation. Under the condition of a non-sinusoidal power supply on with the third harmonic current injected, it is necessary to extend the traditional coordinate transformation of the model for the fifteen-phase induction motor. Based on the traditional coordinate transformation theory, the third harmonic reference coordinate system $d_{\mathrm{t}} q_{\mathrm{t}} 0$ is added to the fundamental reference coordinate system $d_{1} q_{1} 0$ to form a new reference coordinate system $d_{1} q_{1} d_{\mathrm{t}} q_{\mathrm{t}} 0$ and to obtain a transformation matrix of the fundamental and third harmonic interaction. The relationship of the transformation from the abc coordinate system to the $d_{1} q_{1} d_{\mathrm{t}} q_{\mathrm{t}} 0$ coordinate system can be expressed as:

$$
\begin{aligned}
& {\left[\begin{array}{lllll}
f_{\mathrm{ds} 1} & f_{\mathrm{qs} 1} & f_{\mathrm{dst}} & f_{\mathrm{qst}} & f_{0 \mathrm{~s}}
\end{array}\right]^{\mathrm{t}}} \\
& =\mathbf{C}_{d_{1} q_{1} d_{t} q_{t} 0(\theta)}^{a b c}\left[\begin{array}{lllll}
f_{\mathrm{a} 1} & f_{\mathrm{b} 1} & \cdots & f_{\mathrm{d} 3} & f_{\mathrm{e} 3}
\end{array}\right]^{\mathrm{t}}
\end{aligned}
$$

In the above expression, $f$ represents the motor current, voltage or magnetic linkage. In view of the structure of the motor, the matrix of the transformation in (12) can be defined as:

$$
\mathbf{C}_{d_{1} q_{1} d_{t} q_{t} 0(\theta)}^{a b c}=\left[\begin{array}{lll}
\mathbf{C}_{\mathbf{1 1}} & \mathbf{C}_{\mathbf{2 2}} & \mathbf{C}_{\mathbf{3 3}}
\end{array}\right]
$$

$$
\mathbf{C}_{\mathrm{ii}}=\frac{2}{5}\left[\begin{array}{ccccc}
\cos \left[\theta-\frac{\pi}{15}(i-1)\right] & \cos \left[\theta-\frac{2 \pi}{5}-\frac{\pi}{15}(i-1)\right] & \cos \left[\theta-\frac{4 \pi}{5}-\frac{\pi}{15}(i-1)\right] & \cos \left[\theta+\frac{4 \pi}{5}-\frac{\pi}{15}(i-1)\right] & \cos \left[\theta+\frac{2 \pi}{5}-\frac{\pi}{15}(i-1)\right] \\
-\sin \left[\theta-\frac{\pi}{15}(i-1)\right] & -\sin \left[\theta-\frac{2 \pi}{5}-\frac{\pi}{15}(i-1)\right] & -\sin \left[\theta-\frac{4 \pi}{5}-\frac{\pi}{15}(i-1)\right] & -\sin \left[\theta+\frac{4 \pi}{5}-\frac{\pi}{15}(i-1)\right] & -\sin \left[\theta+\frac{2 \pi}{5}-\frac{\pi}{15}(i-1)\right] \\
\cos 3\left[\theta-\frac{\pi}{15}(i-1)\right] & \cos 3\left[\theta-\frac{2 \pi}{5}-\frac{\pi}{15}(i-1)\right] & \cos 3\left[\theta-\frac{4 \pi}{5}-\frac{\pi}{15}(i-1)\right] & \cos 3\left[\theta+\frac{4 \pi}{5}-\frac{\pi}{15}(i-1)\right] & \cos 3\left[\theta+\frac{2 \pi}{5}-\frac{\pi}{15}(i-1)\right] \\
-\sin 3\left[\theta-\frac{\pi}{15}(i-1)\right] & -\sin 3\left[\theta-\frac{2 \pi}{5}-\frac{\pi}{15}(i-1)\right] & -\sin 3\left[\theta-\frac{4 \pi}{5}-\frac{\pi}{15}(i-1)\right] & -\sin 3\left[\theta+\frac{4 \pi}{5}-\frac{\pi}{15}(i-1)\right] & -\sin 3\left[\theta+\frac{2 \pi}{5}-\frac{\pi}{15}(i-1)\right] \\
1 / 2 & 1 / 2 & 1 / 2
\end{array}\right]
$$


Where the sub-matrix $\mathbf{C}_{\mathbf{i i}}(\mathrm{i}=1,2,3)$ is expressed as formula (14), where $\theta$ is the angle between the reference coordinate $\mathrm{d}$-axis rotating at the synchronous rotational angular speed $\omega_{\mathrm{e}}$ and the reference coordinate a-axis of the stationary stator winding.

The application of Formula (13) to Formulas (1) and (8) can result in obtaining the equations of the fifteen-phase motor stator and rotor voltages under the $d_{1} q_{1} d_{\mathrm{t}} q_{\mathrm{t}} 0$ rotating coordinate system.

1) Equations of the Stator Voltage and Magnetic Linkage:

The equations of the motor stator voltage and magnetic linkage are expressed as Formulas (15) and (16):

$$
\begin{gathered}
U_{d q s}=p \psi_{d q s}-\omega A_{d q s} \psi_{d q s}+R_{d q s} I_{d q s} \\
\psi_{d q s}=L_{d q s} I_{d q}
\end{gathered}
$$

Here:

$$
\begin{gathered}
U_{d q s}=\left[\begin{array}{lllll}
u_{d s 1} & u_{q s 1} & u_{d s t} & u_{q s t} & u_{0 s}
\end{array}\right]^{t} \\
\psi_{d q s}=\left[\begin{array}{lllll}
\psi_{d s 1} & \psi_{q s 1} & \psi_{d s t} & \psi_{q s t} & \psi_{0 s}
\end{array}\right]^{t} \\
I_{d q s}=\left[\begin{array}{lllll}
i_{d s 1} & i_{q s 1} & i_{d s t} & i_{q s t} & i_{0 s}
\end{array}\right]^{t} \\
R_{d q s}=\operatorname{diag}\left[\begin{array}{lllll}
r_{s 1} & r_{s 1} & r_{s 1} & r_{s 1} & r_{s 1}
\end{array}\right]^{t} \\
I_{d q r}=\left[\begin{array}{lllll}
i_{d r 1} & i_{q r 1} & i_{d r t} & i_{q r t}
\end{array}\right]^{t} \\
I_{d q}=\left[\begin{array}{lll}
I_{d q s} & I_{d q r}
\end{array}\right]^{t} \\
A_{d q s}=\operatorname{diag}\left[\begin{array}{llll}
A_{2 \times 2} & 3 A_{2 \times 2} & 0
\end{array}\right] \\
L_{d q s}=\left[\begin{array}{llll}
L_{s(5 \times 5)} & \left(L_{m(4 \times 4)}\right. & 0)^{t}
\end{array}\right]
\end{gathered}
$$

$A_{2 \times 2}$ in (23), refers to Formula (25), $L_{s(5 \times 5)}$ and $L_{m(4 \times 4)}$ in (24), can be expressed by formulas (26) and (27), respectively.

$$
\begin{gathered}
A_{2 \times 2}=\left[\begin{array}{ll}
0 & 1 \\
-1 & 0
\end{array}\right] \\
L_{s(5 \times 5)}=\operatorname{diag}\left[l_{l s 1}+3 l_{m 1} l_{l s 1}+3 l_{m 1} l_{l s t}+3 l_{m t} l_{l s t}+3 l_{m t} l_{s 0}\right] \\
L_{m(4 \times 4)}=\operatorname{diag}\left[l_{m 1} l_{m 1} l_{m t} l_{m t}\right]
\end{gathered}
$$

2) Equations of the Rotor Voltage and Magnetic Linkage:

The equations of the motor rotor voltage and magnetic linkage are shown in formulas (28) and (29).

$$
\begin{gathered}
0=p \psi_{d q r}-\left(\omega-\omega_{r}\right) A_{d q r} \psi_{d q r}+R_{d q r} I_{d q r} \\
\psi_{d q r}=L_{d q r} I_{d q}
\end{gathered}
$$

Where:

$$
\begin{gathered}
\psi_{d q r}=\left[\begin{array}{llll}
\psi_{d r 1} & \psi_{q r 1} & \psi_{d r t} & \psi_{q r t}
\end{array}\right]^{T} \\
A_{d q r}=\operatorname{diag}\left[\begin{array}{llll}
A_{2 \times 2} & 3 A_{2 \times 2}
\end{array}\right] \\
R_{d q r}=\operatorname{diag}\left[\begin{array}{llll}
r_{r 1} & r_{r 1} & r_{r t} & r_{r t}
\end{array}\right] \\
I_{d q r}=\left[\begin{array}{llll}
i_{d r 1} & i_{q r 1} & i_{d r t} & i_{q r t}
\end{array}\right]^{T}
\end{gathered}
$$

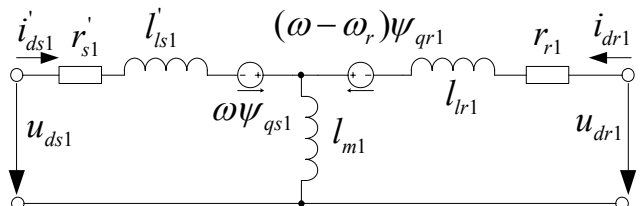

(a)

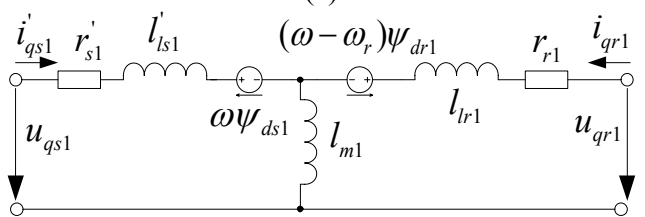

(b)

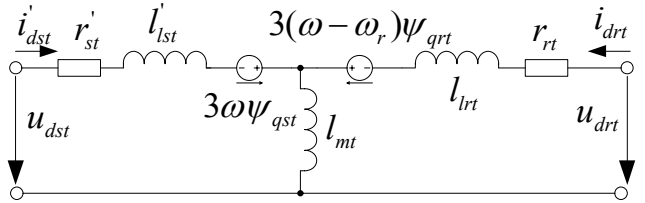

(c)

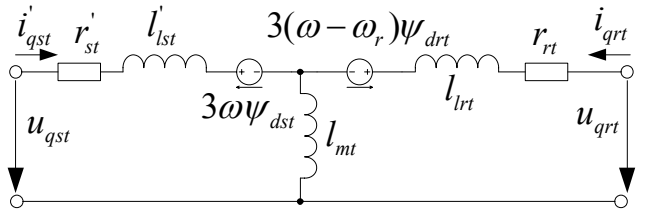

(d)

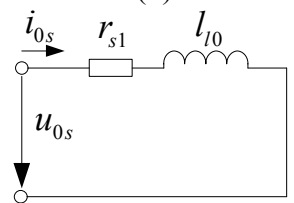

(e)

Fig. 14. The equivalent circuit of the motor fundamental and third harmonic voltage equation on dq axes. (a) The equivalent circuit of $\mathrm{d}$ axes fundamental voltage equation. (b) The equivalent circuit of $\mathrm{q}$ axes fundamental voltage equation. (c) The equivalent circuit of $d$ axes third-harmonic voltage equation. (d) The equivalent circuit of $\mathrm{q}$ axes third-harmonic voltage equation. (e) The equivalent circuit of zero-sequence voltage equation.

$$
L_{d q r}=\left[\begin{array}{lll}
3 L_{m(4 \times 4)} & 0 & L_{r(4 \times 4)}
\end{array}\right]
$$

$L_{m(4 \times 4)}$ in (34) is shown as (27), and $L_{r(4 \times 4)}$ is expressed as follows:

$$
L_{r(4 \times 4)}=\operatorname{diag}\left[l_{l r 1}+l_{m 1} \quad l_{l r 1}+l_{m 1} \quad l_{l r t}+l_{m t} \quad l_{l r t}+l_{m t}\right]
$$

Where $l_{l s 1}$ is the fundamental mutual leakage inductance of the resistance winding, $l_{l r 1}$ is the equivalent fundamental leakage inductance of the rotor, $l_{m 1}$ is the fundamental magnetizing inductance, $r_{s}$ is the stator resistance, and $r_{r 1}$ is the equivalent fundamental resistance of the rotor. (Accordingly, the subscript containing $t$ indicates the parameter of the corresponding third harmonic)

3) Equivalent Circuit of the Motor Stator and Rotor Equivalent Circuit:

Assuming $\quad i_{d s 1}^{\prime}=3 i_{d s 1}, \quad i_{q s 1}^{\prime}=3 i_{q s 1}, \quad r_{s 1}^{\prime}=r_{s 1} / 3$, 
$l_{l s 1}^{\prime}=l_{l s 1} / 3, \quad i_{d s t}^{\prime}=3 i_{d s t}, \quad i_{q s t}^{\prime}=3 i_{q s t}, \quad r_{s t}^{\prime}=r_{s t} / 3, \quad$ and $l_{l s t}^{\prime}=l_{l s t} / 3$, the equivalent circuit of the motor stator, the rotor voltage and the magnetic linkage in the equations can be shown as Fig. 14, where (a)-(b) of Fig. 14 illustrate the equivalent circuit of the motor fundamental voltage equation, (c)-(d) illustrate the equivalent circuit of the motor third harmonic voltage equation, and (e) illustrates the equivalent circuit of the motor zero-sequence voltage equation under the dq coordinate system.

From the equivalent circuit of the motor dq-axis voltage or from the equation of the stator, rotor and magnetic linkage derived above, it can be seen that the fundamental and third harmonic equations are completely decoupled, which can be regarded as two induction motor systems, or the $\omega_{\mathrm{e}}$ and $3 \omega_{\mathrm{e}}$ rotation coordinates. As a result, the orders of the advanced fifteen-phase induction motor system can be greatly simplified.

According to the basic vector relationship of the magnetic-field-oriented indirect control, the synchronous angular frequency $\omega_{\mathrm{e}}$ needs to meet the equation $\omega_{\mathrm{e}}=\omega_{\mathrm{re}}+\omega_{\mathrm{sl}}$ ( $\omega_{\mathrm{re}}$-rotor angular frequency; $\omega_{\mathrm{sl}}$-slip angular frequency). The excitation component $i_{d s 1}$ of the stator current is oriented to the $\mathrm{d}$-axis, and the torque component $i_{q s 1}$ of the stator current is oriented to the q-axis $\left(\psi_{q r}=0, \psi_{d r}=\psi\right)$ to carry out the decoupling control. The equations of the vector control magnetic linkage, slip and torque of the fifteen-phase induction motor are expressed in formulas (36)-(38), respectively.

$$
\begin{gathered}
\psi_{d r 1}=3 l_{\mathrm{m} 1} i_{d s 1} \\
\omega_{\mathrm{s} 1}=\frac{3 l_{\mathrm{m} 1}}{T_{\mathrm{r} 1} \psi_{d r 1}} i_{q s 1} \\
T_{\mathrm{m} 1}=\frac{5}{2} P\left(\frac{l_{\mathrm{m} 1}}{l_{\mathrm{r} 1}} i_{d s 1} \psi_{d r 1}\right)
\end{gathered}
$$

\section{BLOCK DIAGRAM OF THE MOTOR CLOSED-LOOP VECTOR CONTROL}

Based on the above derivation, the block diagram for the closed-loop vector control of the fifteen-phase PWM driver is shown in Fig. 15.

There exists a direct relationship between the air-gap magnetic density and the port voltage application in the motor. The approximate control of the air-gap magnetic density of the motor can be conducted simply and directly by an open-loop adjustment of the phase and amplitude relationship between the fundamental modulation wave and the third-harmonic modulation wave. Because the unfixed relationship between the air-gap magnetic density and the port voltage varies with the load power, the phase relationship between the corresponding fundamental modulation wave and the thirdharmonic modulation wave need a real-time adjustment according to the load power. The size of the load power can be

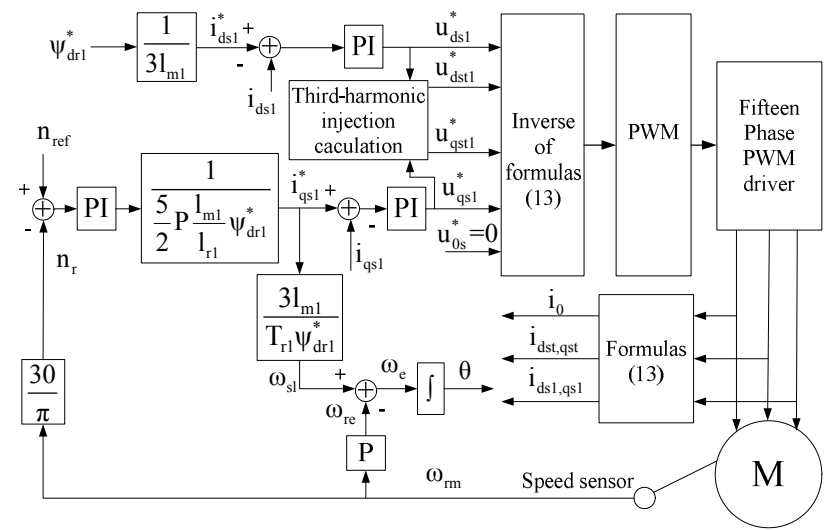

Fig. 15. The closed-loop vector control of the fifteen-phase PWM driver.

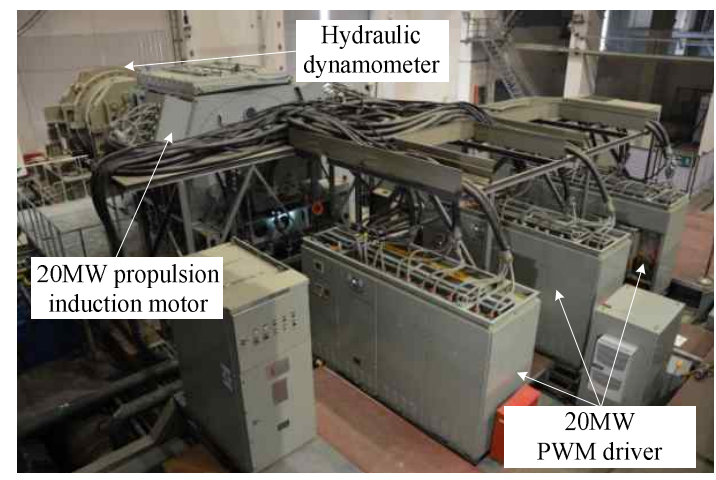

Fig. 16. The $20 \mathrm{MW}$ fifteen-phase PWM driver on the test platform.

estimated according to the torque current magnitude and speed. The phase relationship needed for injection can be obtained through off-line calculations beforehand and through a look-up table at the time of control.

\section{THE EXPERIMENT RESULTS}

In order to demonstrate the performance of the 20MW fifteen-phase PWM adjustable-speed driver, various kinds of comprehensive experiments have been made by loading a 20MW fifteen-phase propulsion motor in terms of control strategy and power capacity. The switching frequency of the fifteen-phase PWM driver is $500 \mathrm{~Hz}$. Fig. 16 shows a photo of the developed 20MW PWM driver on a test platform that is attached to an advanced 20MW fifteen-phase propulsion induction motor for a test under a full power load. The prototype of the PWM driver is 18 cubic meters in volume, and it is less than 15 tons in weight. The main topology of the PWM driver is shown in Fig. 3. Its input voltage is provided by a 20MW 12-phase rectifier generator. The load of the PWM driver is an advanced 20MW fifteen-phase propulsion induction motor adopting a pattern of evaporative cooling. The load of the propulsion motor is a hydraulic dynamometer, with its power and cubic speed being linear. 


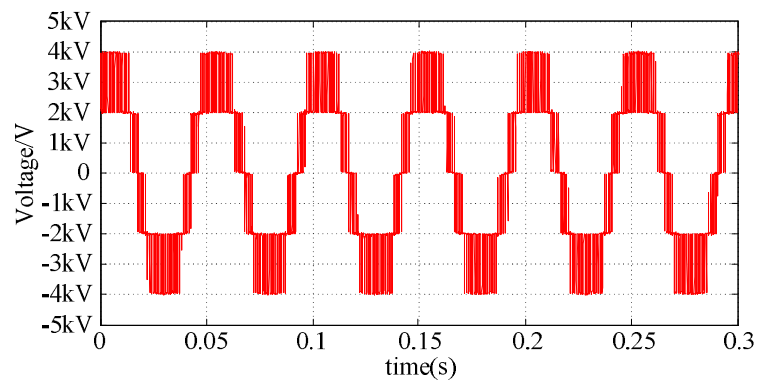

Fig. 17. Phase voltage waveforms of single-channel in PWM driver under 20MW full-load operating conditions.

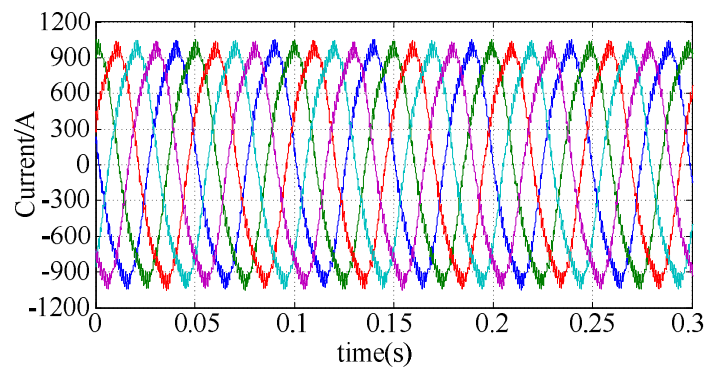

Fig. 18. Five-phase current waveforms of single-channel in PWM driver under 20MW full-load operating conditions.

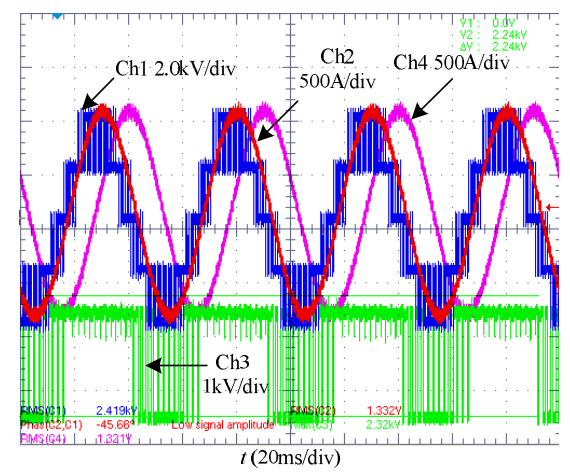

Fig. 19. The waveforms of phase current, voltage and switch voltage under 20MW full-load operating conditions (Ch1: Blue represents, A1-phase voltage; Ch2: Red represents, the load current of A1-phase; Ch3: Green represents, the voltage of inner switch; Ch4: Purple represents, the load current of B1- phase).

\section{A. 20MW Full Power Test}

The single-channel voltage and current waveforms of the PWM driver attached to a fifteen-phase propulsion motor under the 20MW full-power operating conditions are shown below. At this time, the speed of the propulsion motor is rated at 200/RPM, and the injected third harmonic voltage is one eighth of the rated fundamental voltage. The output voltage and current waveforms of the single-channel (one phase voltage and five phase current) in the PWM driver are shown in Fig. 17 and Fig.18, respectively. The A1-phase current, A1-phase voltage, B1-phase current and the inner switch voltage waveforms of the first channel in the PWM driver are shown in Fig. 19.

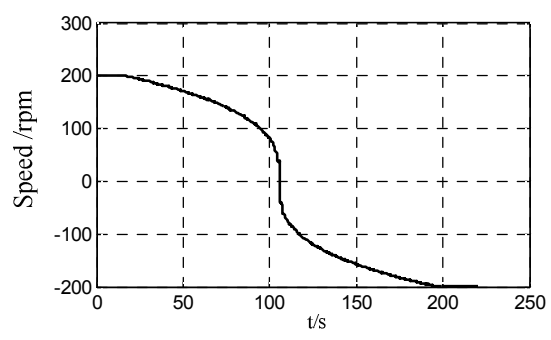

(a)

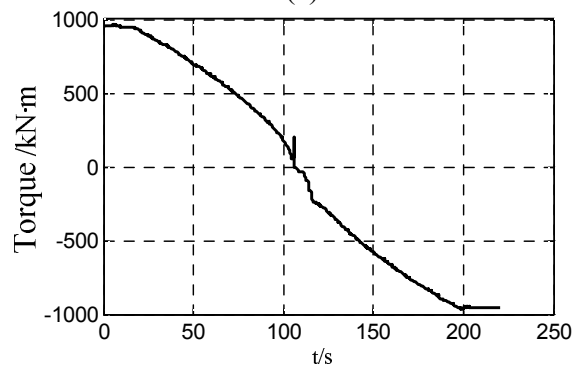

(b)

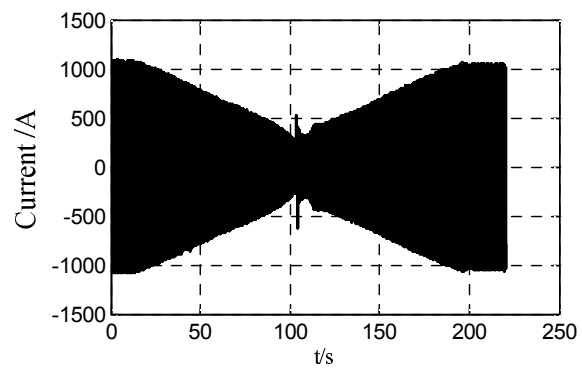

(c)

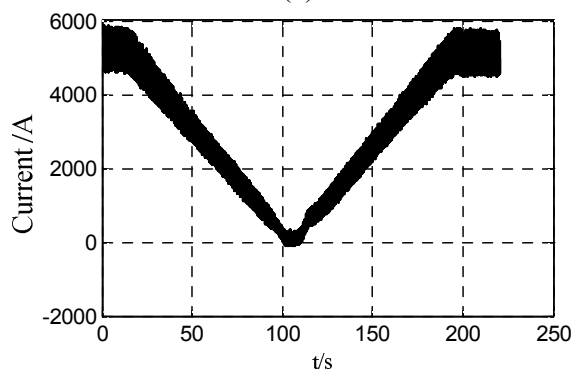

(d)

Fig. 20. The correlated test waveforms of forward-backward rotation process with propulsion motor on-load. (a) The rotation speed curve of propulsion motor. (b) The torque curve of hydraulic dynamometer. (c) The A1 phase current envelope of PWM driver. (d) The input DC current curve of PWM driver.

\section{B. Forward and Backward Rotation Test}

In view of a linear exiting between the cube of the rotational speed of the propeller and the output power of the propulsion motor when a real ship is running, a hydraulic dynamometer is used to simulate the linear relationship at the test site. Since the power and speed form a cubic relationship, the fact that the demand for the gas turbine power increases linearly with time should be taken into account. For this reason, the speed command is given by the cube of time. With a square relationship between the torque and the speed of the hydraulic 
dynamometer, the torque and the instruction time of the rotating speed is two thirds of the power relationship.

As shown in Fig. 20, the test waveforms appear when the new 20MW fifteen-phase induction propulsion motor turns from positive 200RPM to negative 200RPM. Fig. 20(a) shows the speed curve of the forward and backward rotation of the propulsion motor with a load. It can be seen that the rotating speed and time form an approximate open square relationship. Fig. 20(b) shows the torque curve of the hydraulic dynamometer. It can be seen that the torque changing with time is two thirds of the power relationship. Fig. 20(c)-(d) show the envelope curve of the A1 phase and the input DC current curve of the PWM driver when the PWM driver loads the propulsion motor forward and backward. It can be seen that the motor changes smoothly from forward to backward and vice versa. The current mutation in the envelope curves at the change of speed shows the rapid adjustment capability of the PWM driver torque current inner loop control. This test has achieved the purpose of simulating the characteristics of the propeller load.

\section{10MW Abrupt Unloading Test}

In bad weather, the propeller will repeatedly rise from and sink into water, so that the corresponding propulsion motor shaft power will load and unload suddenly. Under this working condition, the demand for the dynamic performance of the drive is higher. Therefore, an abrupt unloading test has been conducted on the 20MW fifteen-phase propulsion motor with a $10 \mathrm{MW}$ load. In this test, the propulsion motor driven by the control inverter ran stably under the operating conditions of $50 \%$ power at the rated speed. At this time, the coaxial propulsion motor was loaded with a $5 \mathrm{MW}$ DC generator and a 5MW hydraulic dynamometer. Then, under a unified command, the DC generator load switch and the hydraulic dynamometer water pump inverter switch were disconnected at the same time. Then the 5MW DC generator load was disconnected instantaneously. However, the 5MW hydraulic dynamometer experienced a relatively slow unloaded process due to the inertia of the water flow in the pipeline. This situation is similar to that of the propeller rising from and sinking into water. Hence, it is possible to make an approximate simulation of the abrupt unloading during high waves and winds.

Fig. 21(a) shows the partial phase current envelope waveform of the PWM driver under the condition of a $10 \mathrm{MW}$ abrupt unloading. Fig. 21(b) shows a detailed drawing of the phase current waveform of the PWM driver under the condition of a 10MW abrupt unloading. Fig. 21(c) shows the curve of the excitation current, torque current and zero sequence current recorded by the man-machine interface of the PWM driver. Fig. 21(d) gives the details of these three currents. The abrupt unloading point of the 5MW water-cooled dynamometer machine and that of the $2.5 \mathrm{MW}$ DC generator are the records of the two system diagnostic tests which were conducted before the integral $10 \mathrm{MW}$ abrupt unloading test.

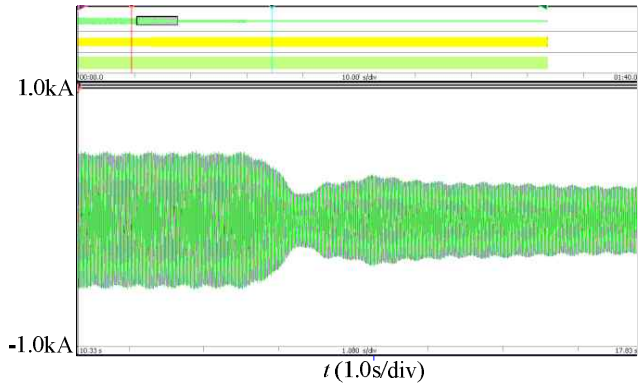

(a)

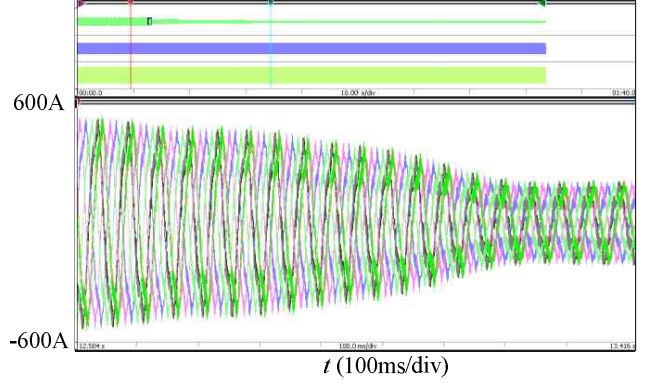

(b)

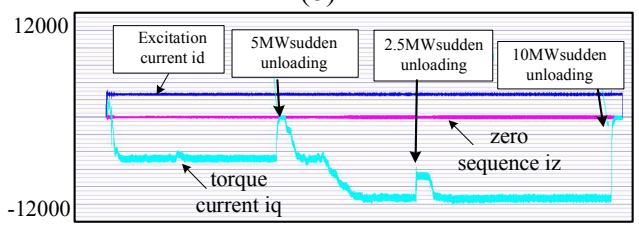

(c)

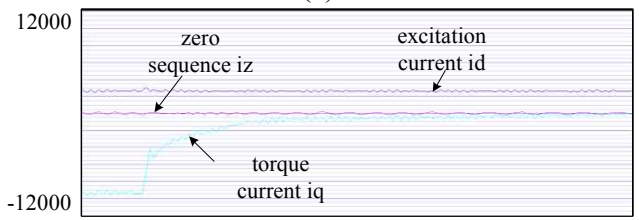

(d)

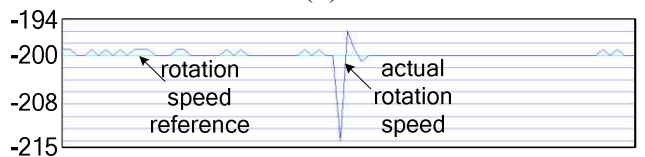

(e)

Fig. 21. The experiment waveforms under the condition of $10 \mathrm{MW}$ abrupt unloading. (a) The phase current envelope of PWM driver. (b) The phase current waveforms of PWM driver in detail. (c) The diagram of excitation current, torque current and zero-sequence current. (d) The detail drawing of exciting current, torque current and zero-sequence current. (e) The curves of the motor speed reference and actual motor speed.

Fig. 21(e) shows a chart of a comparison between the speed command and the actual motor speed under the condition of a $10 \mathrm{MW}$ abrupt unloading. The phase current waveform of the motor is relatively stable without large sudden changes under the condition of 10MW abrupt unloading. This is because of the correct control functions and fast dynamic response. At the instant when a $10 \mathrm{MW}$ abrupt unloading occurs, the motor speed overshoots $7 \%$ (14RPM) and is quickly stabilized at the -200RPM set by the instructions through a relatively rapid adjustment. 


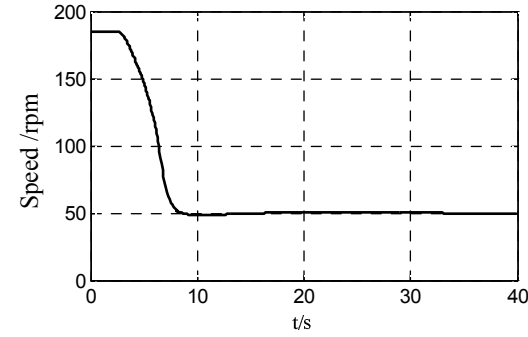

(a)

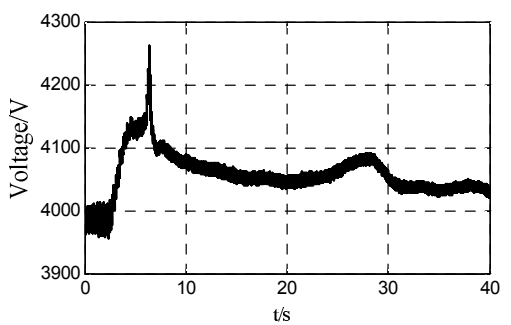

(b)

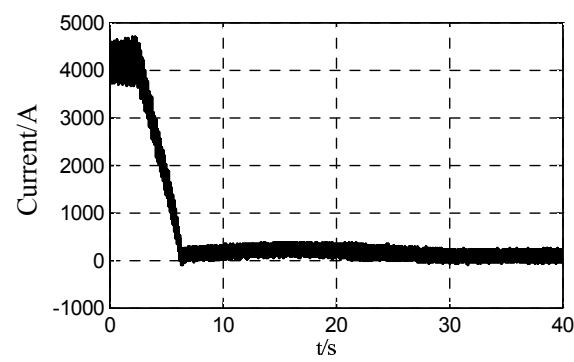

(c)

Fig. 22. The correlated speed-regulating performance test waveforms of PWM driver. (a) The actual speed curve of propulsion motor. (b) The input DC voltage curve of PWM driver. (c) The input DC current curve of PWM driver.

\section{Rapid Speed Adjustment Test}

The rapid speed adjustment of propulsion motors is vital to improvements in the safety, flexibility and combat performance of ships. Fig. 22 shows the test waveforms of the propulsion motor when the rotation speed decreases from 185/rpm (approximately $16 \mathrm{MW}$ load) to 50/rpm (approximately $0.3 \mathrm{MW}$ load) within 4 seconds. Fig. 22(a) shows the actually measured motor speed waveform. Fig. 22(b)-(c) show the input DC voltage and current waveforms of the PWM driver, respectively. From Fig. 22 it is found that the whole speed-down process is rapid, smooth and stably controllable. An increase in the DC input voltage of the PWM driver has resulted from the power feedback regenerated by the motor. When the DC bus voltage reaches the threshold voltage that causes the brake unit to work, the brake unit will ensure that the DC input voltage of the PWM driver will not be high enough to affect the safe operation of the driver.

\section{CONCLUSIONS}

The paper has given a detailed description of the system structure of a 20MW PWM driver for an advanced fifteen-phase propulsion induction motor with a special third-harmonic injection. The modular design method is adopted for the main circuit hardware to avoid the larger switch stress caused by the multiple turn on of the IGBTs in conventional large-capacity converter systems. In addition, the safety of system is improved. Moreover, based on $125 \mathrm{Mbps}$ high-speed fiber-optic ring net communication, the distributed control of the PWM driver is achieved, which results in a simplified control circuit, improved reliability and maintainability of the control subsystem. An analytical model of the fifteen phase induction motor is deduced. Then, the indirect rotor field oriented vector control algorithm is obtained. Finally, the design method and analytical model are verified by static and transient tests. The developed 20MW fifteen-phase PWM driver for propulsion motors can be used for electrical propulsion in large commercial ships and surface ships.

\section{ACKNOWLEDGMENT}

This work was supported by the National Natural Science Foundation of China (NSFC) under Grant 51177170.

\section{REFERENCES}

[1] A. Crane and T. J. McCoy, "Electromagnetic compatibility design for a 19 MW PWM motor drive," IEEE Industry Applications Conference, Vol. 3, pp. 1590-1595, 1999.

[2] P. Manuelle, B. Singam, and S. Siala, "Induction motors fed by PWM MV7000 converters enhance electric propulsion performance," Power Electronics and Applications, EPE '09. 13th European Conference, pp. 1-9, 2009.

[3] M. D. Bogomolov, A. Borisavljevic, and E. A. Lomonova "Concept study of $20 \mathrm{MW}$ high speed permanent magnet synchronous motor for marine propulsion," $\mathrm{PhD}$. Thesis, Eindhoven University of Technology, Sep. 2013.

[4] E. Levi, R. Bojoi, and H. A. Toliyat, "Multiphase induction motor drives - a technology status review," IET Electric Power Applications, Vol. 1, No. 4, pp. 489-516, Jul. 2007.

[5] M. Sowmiya, G. Renukadevi, and K. Rajambal, "IFOC of a five-phase induction motor drive," Power, Energy and Control (ICPEC), 2013 International Conference on, Sri Rangalatchum Dindigul, pp. 304-309, 2013.

[6] A. S. Abdel-Khalik, S. Ahmed, A. A. Elserougi, A. M. Massoud, "A voltage-behind-reactance model of five-phase induction machines considering the effect of magnetic saturation," IEEE Trans. Energy Convers., Vol. 28, No. 3, pp. 567-592, Sep. 2013.

[7] H. S. Che, E. Levi, M. Jones, W.-P. Hew, and N. A. Rahim, "Current control methods for an asymmetrical six-phase induction motor drive," IEEE Trans. Power Electronics, Vol. 29, No. 1, pp. 407-417, Jan.2014.

[8] A. Taheri, A. Rahmati, and S. Kaboli, "Efficiency improvement in DTC of six-phase induction machine by adaptive gradient descent of flux," IEEE Trans. Power Electron., Vol. 27, No. 3, pp. 1552-1562, Mar. 2012. 
[9] P. G. Sharma1 and S. Rangari, "Simulation of inverter fed five phase induction motor," International Journal of Science and Research, Vol. 2, No. 2, pp. 127-132, Feb. 2013.

[10] J. A. Riveros, M. J. Duran, F. Barrero, and S. Toral, "Direct torque control for five-phase induction motor drivers with reduced common-mode voltage," IECON 2012-38th Annual conference on IEEE industrial electronics society, pp. 3616-3621, 2012.

[11] S. Senthilkumar and S. Vijayan, "Simulation of high performance PID controller for induction motor speed control with mathematical modeling," Research Journal of Applied Sciences Engineering and Technology, Vol. 6, No. 18, pp. 3343-3348, Feb. 2013.

[12] B. Purwahyudi, Saidah, and H Afianti, "Fuzzy logic controller for Volts/Hz induction motor control used in electrically driver maring propeller," Academic Research International, Vol. 4, No. 5, pp. 258-265, Sep. 2013.

[13] C. S. Lim, E. Levi, M. Jones, N. A. Rahim, and W. P. Hew, "FCS-MPC-based current control of a five-phase induction motor and its comparison with PI-PWM control," IEEE Trans. Ind. Electron., Vol. 61, No. 1, pp. 149-163, Jan. 2014.

[14] N. R. Abjadi, A. M. Gholamreza, and S. Jafar, "Model following sliding-mode control of a six-phase induction motor driver," Journal of Power Electronics, Vol. 10, No. 6, pp. 694-701, Nov. 2010

[15] D. Wang, X. Z. Wu, Y. J. Guo, and J. Q. Chen, "Electromotive force calculation of fifteen-phase induction motors with non-sinusoidal supply," in Proc. the CSEE, Vol. 33, No. 9, pp. 129-137, Mar. 2013. (in Chinese)

[16] D. Wang, X. Z. Wu, Y. J. Guo, and J. Q. Chen, "Determination of harmonic voltages for fifteen-phase induction motor with non-sinusoidal supply," in Proc. the CSEE, Vol. 32, No. 24, pp. 126-133, Aug. 2012. (in Chinese)

[17] D. Wang, W. M. Ma, Y. J. Guo, D. Liu, and J. Chen, "Modelling of multiphase induction motor with non-sinusoidal supply," Trans. China Electrotechnical Society, Vol. 25, No. 2, pp. 6-14, Feb. 2010. (in Chinese)

[18] W. M. Ma, "Development of vessel integrated power system," Electrical Machines and Systems (ICEMS), pp.1-12, 2011.

[19] D. Giulivo, G. Sulligoi, and A. Tessarolo, "Electric motors and drives for modern ship thruster propulsion: Design and system integration issues," Electrical Systems for Aircraft, Railway and Ship Propulsion (ESARS), pp. $1-6,2010$

[20] M. M. Bakran, M. Helsper, and H. G. Eckel, "Multiple turn on of IGBTs in large inverters," IEEE Industrial Electronics, IECON 2006, 32nd Annual Conference, pp. 1609-1614, 2006

[21] M. M. Bakran, G. Eckel, H. M. Helsper, and A. Nagel, "Next generation of IGBT-modules applied to high power traction," Power Electronics and Applications, 2007 European Conference on. IEEE, Aalborg, pp. 1-9, 2007.

[22] A. Tani, M. Mengoni, L. Zarri, G. Serra, and D. Casadei, "Control of multiphase induction motors with an odd number of phases under open-circuit phase faults," IEEE Trans. Power Electron., Vol. 27, No. 2, pp. 565-577, Feb. 2012.

[23] A. S. Nanoty and A. R. Chudasama, "Design of multiphase induction motor for electric ship propulsion,"
Electric Ship Technologies Symposium (ESTS), IEEE, pp. 283-287, 2011

[24] C. Zhang, W. M. Ma, and C. Sun, "A switchable high-speed fiber-optic ring net topology and its method of high-performance synchronization for large-capacity power electronics system," International Journal of Electrical Power \& Energy Systems, Vol. 57C, pp. 335-349, May. 2014.

[25] F. Jerry, G. Jinghong, and H. Stephen, "Protocol design of dual ring PESNet(DRPESNet)," CPES 2002 power electronics seminar, pp. 1301-1306, 2002.

[26] C. Sun, C. Zhang, and S. Ai, "Topology and protocol of practical high-speed fiber-optic ring net for large-capacity power electronic systems," in Proc. the CSEE, Vol. 32, No. 15, pp. 63-73, 2012. (in Chinese)

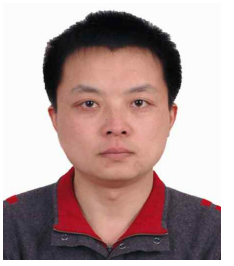

Chi Sun was born in 1977. He received his M.S. degree in Power Electronic and Electric Drive at the Radar Institute of Air Force, Wuhan, China, in 2001, and his Ph.D. degree in Project of Weapon System and Application at the Ordnance Engineering College, Shijiazhuang, China, in 2004. Dr. Sun is now a Professor and Supervisor for $\mathrm{Ph}$.D. candidates with NUE, Wuhan, China. His current research interests include medium-high voltage and large-capacity power electronic converter technology and control-related technology.

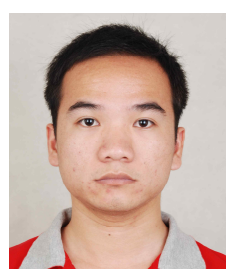

Sheng Ai was born in Wuhan, China, in 1985. He received his B.S. and M.S. degrees in Electrical Engineering from NUE, Wuhan, China, in 2007 and 2009, respectively. He is presently working toward his Ph.D. degree. His current research interests include medium-high voltage and large-capacity power electronic converter technology.

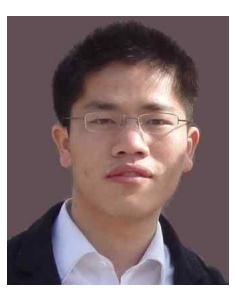

Liangdeng Hu was born in Xiangtan City of Hunan Province in 1986. He received the B.S. and M.S. degree in electrical engineering from NUE, Wuhan, China, in 2008 and 2010, respectively. He is currently working toward the Ph.D. degree. His researches are currently focused on medium-high voltage and large-capacity power electronic converter technology as well as high power IGBT digital gate drive technology.

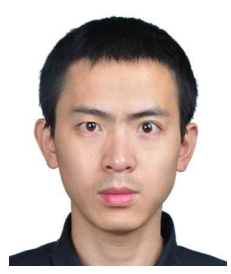

Yulin Chen was born in Baoding City of Hebei Province in 1986. He received the B.S. and M.S. degree in electrical engineering from NUE, Wuhan, China, in 2009 and 2012 , respectively. He is currently working toward the Ph.D. degree. His researches are currently focused on medium-high voltage and large-capacity power electronic converter technology as well as high power IGBT digital gate drive technology. 